\title{
Diagnosis and Outcome of Tuberculosis of Knee Joint (Gonitis Tuberculosis) with Pulmonary Tuberculosis after Completing Anti-Tuberculosis Therapy: A Case Report
}

\author{
Anita Nur Charisma*, Winariani Koesoemoprodjo \\ Department of Pulmonology and Respiratory Medicine, Faculty of Medicine, Universitas Airlangga/Dr. Soetomo \\ General Hospital, Surabaya, Indonesia.
}

\section{ARTICLE INFO}

\section{Article history:}

Received 09 October 2020

Received in revised form 08 January 2021

Accepted 19 January 2021

Available online 30 January 2021

\section{Keywords:}

Knee joint tuberculosis,

Gonitis tuberculosis,

Completed treatment outcome.

\begin{abstract}
Background: Tuberculosis (TB) in extra-pulmonary organs, such as bone and joint TB, has an incidence rate of $19 \%$ of the cases and represents $11-15 \%$ of all extra-pulmonary TB. The predilection of bones and joints is the most common predilection with more than $10 \%$ of the cases. Gonitis TB is a monoarthritis, chronic progressive, and intermittent disease. Hematogenous spreads through synovial or subchondral or as a focus in the juxtaarticular bone. The spread can also occur indirectly from osseous lesions of the epiphyseal bone in adults or metaphysis in children, which causes erosion in the joint space and becomes arthritis.

Case: A 19-year-old female with a chief complaint of swelling in the right knee joint accompanied by pain and difficulty in the legs to move and straighten the body. Another complaint was cough with whitish sputum, fever and night sweating, decreased appetite, and loss of body weight. Radiological chest X-ray showed fibro-infiltrates in both hemithoraces and radiological of the right genu, impression like joint effusion and mass density opacity in the popliteal, and suspicious abscess in the soft tissue. GeneXpert MTB/RIF examination of genu tissue and sputum: medium Mycobacterium tuberculosis (M.Tb) was detected, rifampicin sensitive. Histopathology from the open biopsy showed the tissue consisted of epitheloid-shaped histiocyte cells forming granulomas. Ziehl Neelsen staining of the tissue obtained acid-fast bacillus (AFB).

Conclussion: Gonitis TB is a hematogenous spread of M.Tb from infection with a deep primary focus on the joint that is chronic progressive and generally affects one joint. The management can be done by administering anti-tuberculosis and clinicalmonitoring.
\end{abstract}

\section{INTRODUCTION}

Tuberculosis (TB) is an infectious disease caused by Mycobacterium tuberculosis (M.Tb) that occurs in the pulmonary or extra-pulmonary. The prevalence is 5$15 \%$, or around 1.7 billion people are infected with M.Tb that becomes TB disease. ${ }^{1,2}$ TB in extra- pulmonary organs, such as bone and joint TB, has an incidence rate of $19 \%$ of the cases and represents $11-15 \%$ of all extrapulmonary $\mathrm{TB}, 2,3$ ranked third after pleuritis and lymphadenopathy TB. ${ }^{4}$ The predilection of bones and joints TB in the knee joint (gonitis TB) is the second most common predilection after TB of the spine (spondylitis TB), with more than $10 \%$ of the cases. ${ }^{2,5}$
Gonitis TB is a monoarthritis that generally occurs in one joint, a chronic progressive and intermittent disease in which if not treated well, it will get worse by forming abscess and fistula. ${ }^{6}$ It can generally occur at any age and is common in children and adolescents more than adults. Hematogenous spreads through synovial or subchondral (such as distal femur, proximal tibia, or patella) or as a focus in the juxta-articular bone. The spread can also occur indirectlyfrom osseous lesions of the epiphyseal bone (in adults) or metaphysis (in children), which causes erosion in the joint space and becomes arthritis. ${ }^{4,7}$ 
This work is licensed under a Creative Commons Attribution-Share Alike 4.0 International License. 


\section{CASE}

A 19-year-old female with a chief complaint of swelling in the right knee joint for 6 months and worsened 2 months before admission to the hospital.The swelling occurred after falling and got bigger accompanied by pain and difficulty in the legs to move and straighten the body. Another complaint was cough with whitish sputum for one month, fever and nightsweating for a week, decreased appetite and loss of bodyweight around $10 \mathrm{~kg}(50 \mathrm{~kg}$ to $40 \mathrm{~kg}$ ) for 3 months. From physical examination of the lungs, palpation of the fremitus was decreased in $2 / 3$ lower of the right hemithorax. Percussion was dull in $2 / 3$ lower of the right hemithorax. Vesicular breath auscultation was decreased in $2 / 3$ lower of the right hemithorax, rhonchi in 2/3upper of the right hemithorax. Lower limb examination showed swelling in the right knee joint (genu), pain and limited movement with difficulty to move and straighten the body. Laboratory data: leukocytosis (WBC: 12.33 103/ $\mu 1$ ), thrombocytosis

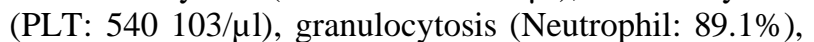
and increased CRP ( $8.4 \mathrm{mg} / \mathrm{dL})$.

Radiological chest X-ray showed dullness in right costophrenic angle towards right pleural effusion, parenchyma contained fibro-infiltrates in both hemithoraces, thoracic ultrasonography (USG) showed that there was an organized right pleural effusion (Figure 1). Radiological X-ray right genu, impression of the joint effusion, mass density opacity in the popliteal, suspicious abscess on soft tissue, lower extremity MRI with contrast showed pyomyositis with right genu synovitis (Figure 2). FNAB USG-guiding examination on distal femur of right genu showed that there were inflammatory lymphocytes, neutrophils, histiocytes, plasma cells, and found necrotic debris and no signs of malignancy; the conclusion was suppurative chronic inflammation. The patient was planned for elective debridement surgery and synovectomy by orthopedic surgery, after previously not improving with analgesic mefenamic acid $500 \mathrm{mg} / 8$ hours (Figure 3).

Synovial fluid examination showed cloudy color with leukocytes 35,000 cells/ul, crystals negative, PMN $25 \%$, MN $98.2 \%$, glucose $25 \mathrm{mg} / \mathrm{dl}$, and total protein 4 gr/dl. GeneXpert MTB/RIF examination of genu tissue: medium M.Tb was detected, rifampicin sensitive, and GeneXpert MTB/RIF sputum: medium M.Tb was detected, rifampicin sensitive. Culture sensitivity sputum found M.Tb and Pseudomonas aeruginosa, Ziehl Neelsen (ZN) staining sputum found acid-fast bacilli(AFB) $2+$.

Histopathology from open biopsy right genu tissue, the macroscopic showed blackish brown with soft consistency. Microscopic showed the tissue consisted of epitheloid-shaped histiocyte cells forming granulomas. Multinucleated giant cells appeared with nuclei lined up to form a "horse-shoe" (datia langhans cells), large necrotic area in between. $\mathrm{ZN}$ staining of tissue obtained AFB. The biopsy revealed granulomatous inflammation according to TB (Figure 4).

The patient was diagnosed with post debridement - synovectomy right knee joint ec gonitis TB, new case of pulmonary TB, community acquired pneumonia (CAP) PSI score 39 class II, and organized right pleural effusion.

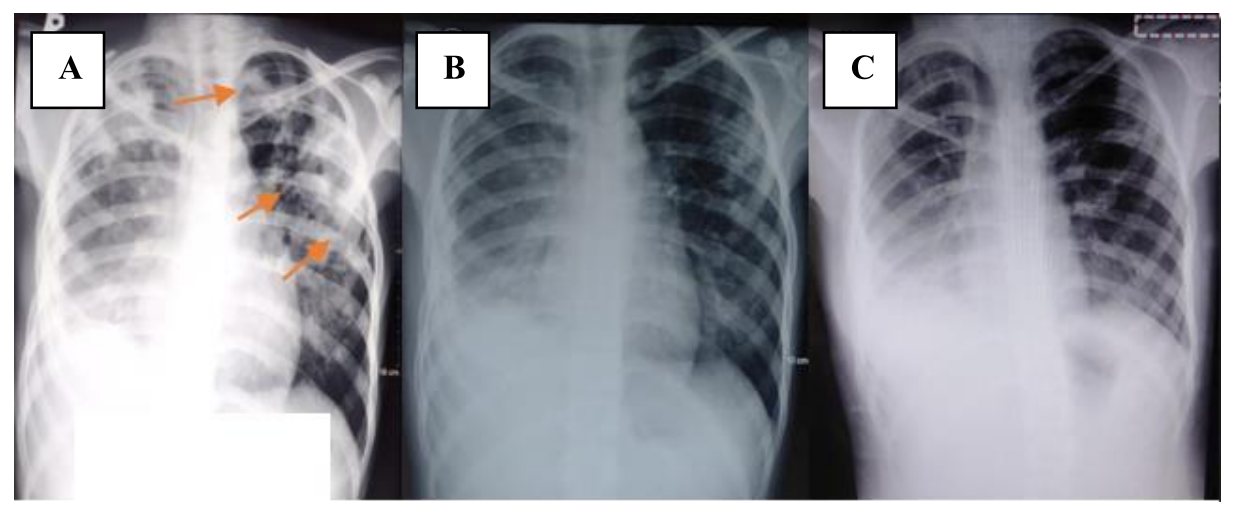

Figure 1. A) Chest X-ray before anti-tuberculosis therapy, showing infiltrates in the right suprahillar, showing fibroinfiltrates in both hemithoraces, dullness in right costophrenic angle, (showing pulmonary TB and right pleural effusion, partially organized),

B) Fibro-infiltrates improvement, after 6 months with anti-tuberculosis therapy,

C) Completed therapy of anti-tuberculosis for 12 months. 

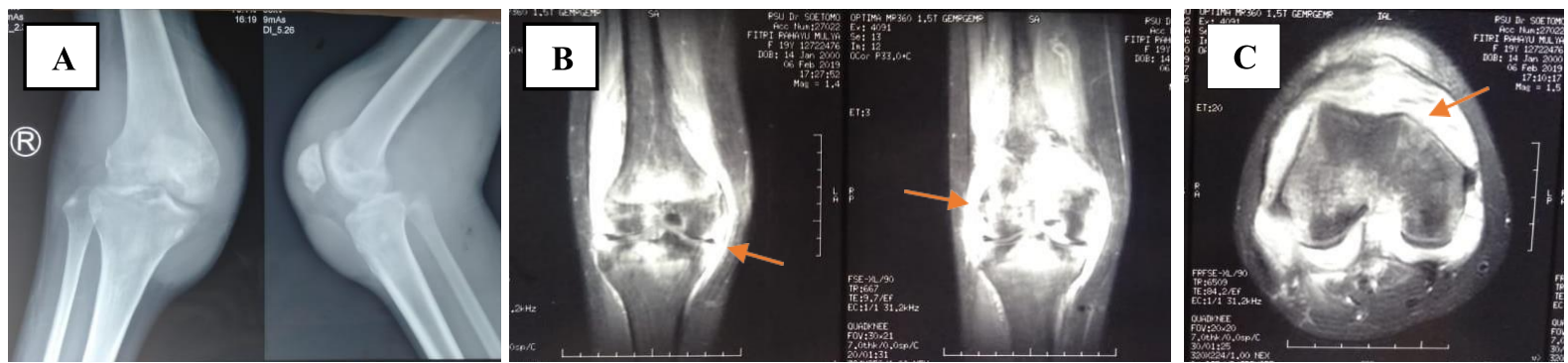

Figure 2. A) Radiological X-ray right genu, impression of joint effusion, and mass density opacity in the poplitea, suspicious of an abscess on the soft tissue. B) MRI of lower extremity with contrast showed visible intramuscular hyperintensity lesion involving m.poplitea, m.vastus intermedius, m.plantaris, m.gastrocnemius, m.satorius and lymph nodes in the popliteal vessel. C) Hypo-intense thickening of the synovium with strading hoffas's fat to the pre- patellar stage. Conclusion of pyomyositis accompanied by synovitis on the right genu, without bone narrow edema (fluid in the bone marrow).
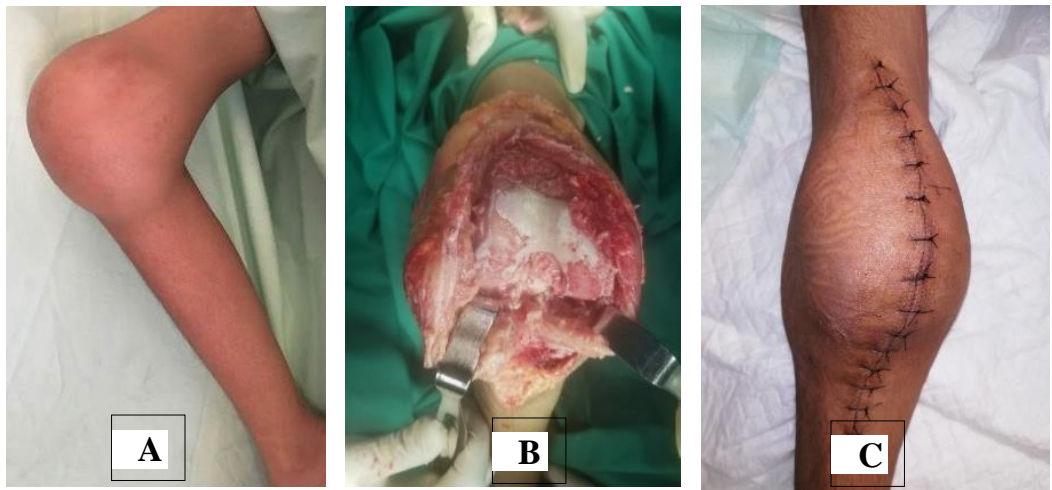

Figure 3. A) Pre-operative debridement - synovectomy right genu from lateral positions. B) Intra-operative found pus accompanied by caseous necrosis tissue. C) Post-operative.

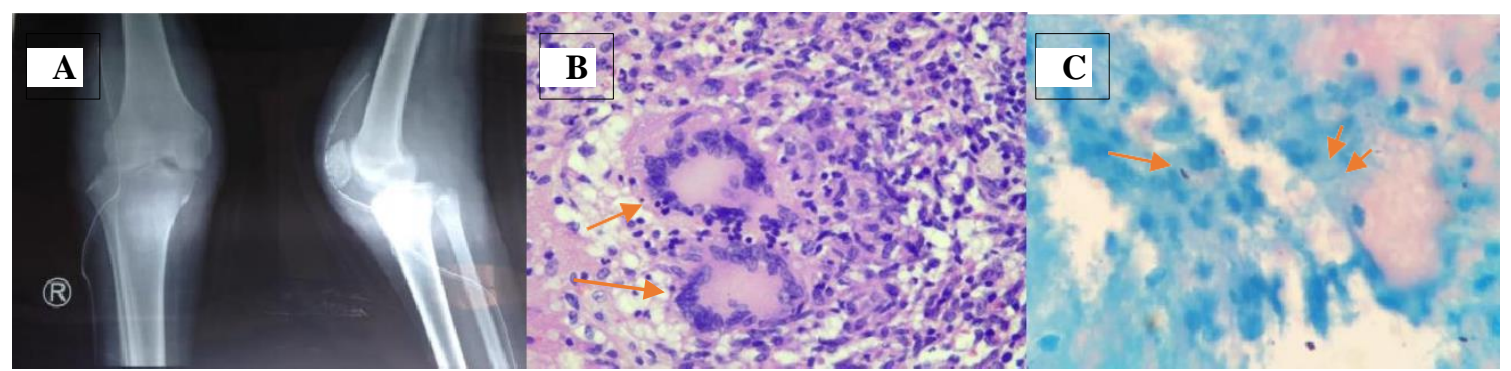

Figure 4. A) Radiological X-ray right genu evaluation post-operative with drainage.

B) Histopathology from open biopsy right genu tissue, microscopic showed the tissue consisted of epitheloid-shaped histiocyte cells forming granulomas. Multinucleated giant cells appeared with nuclei lined up to form a "horse shoe" (datia langhans cells), large necrotic area in between. C) ZN staining of tissue obtained AFB. The biopsy revealed granulomatic inflammation according to TB.

\section{Therapy \& Evaluation}

Therapeutic treatment while in the hospital, the patient was given Ceftriaxone 1 gram/12 hours for Pseudomonas aeruginosa, and based on laboratory evaluation, the leukocytes $12.3310^{3} / \mu 1$ improved to 9.58 $10^{3} \mu 1$. The antibiotics were discontinued after 7 days. In the treatment of gonitis and pulmonary TB, it was given for 9-12 months with anti-tuberculosis category 1 with
HRZE regimen (body weight $40 \mathrm{~kg}$ ): Isoniazid $300 \mathrm{mg}$ every 24 hours, Rifampin $450 \mathrm{mg}$ every 24 hours, Pyrazinamide $1000 \mathrm{mg}$ every 24 hours, and Ethambutol $750 \mathrm{mg}$ every 24 hours during hospitalization. After being discharged from the hospital, the patient continued the therapy with intensive phase anti-tuberculosis category 1 with 4 fixed-dose combinations (FDC) of 3 tablets at the public health center. 


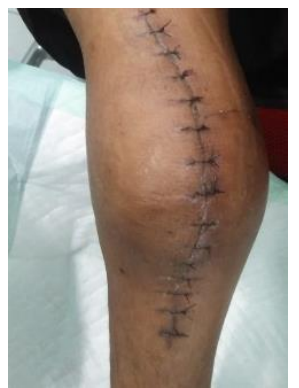

A) First control, 3 days after being discharged

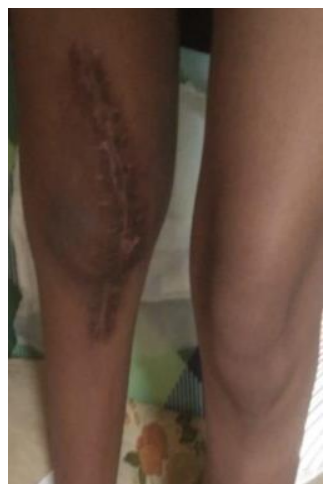

E) 6 months after therapy

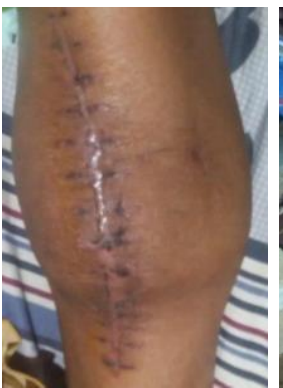

B) Post removal hecting

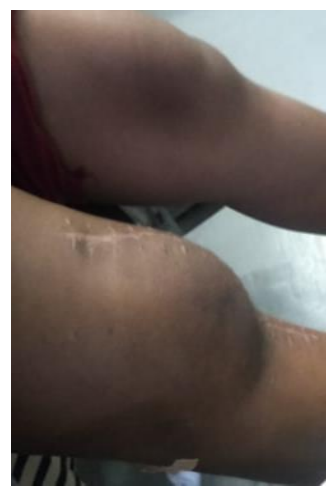

F) 9 months after therapy from anterior and lateral positions

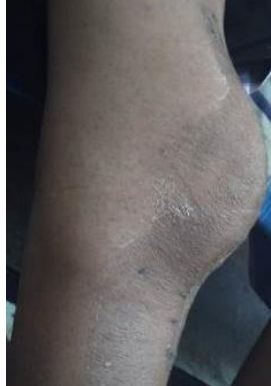

C) 2 months after anti-tuberculosis therapy from anterior and lateral positions

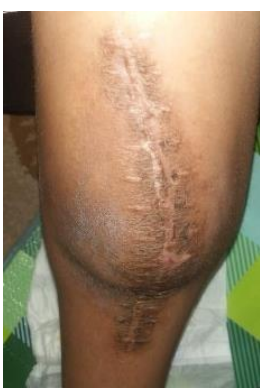

D) 6 months with flexi position

Figures 5. Clinical evaluation of completed anti-tuberculosis therapy.

Evaluation of smear sputum at the end of $2^{\text {nd }}$ month treatment showed the results of AFB conversion to be negative. The patient continued the advanced phase treatment with 3 tablets of 2 FDC. Evaluation of the smear sputum AFB at the end of the $5^{\text {th }}$-month treatment and at the $6^{\text {th }}$ month showed that the results were still negative. Chest $\mathrm{X}$-ray evaluation showed fibro-infiltrate improvement after completing therapy of antituberculosis for 12 months (Figure 1).

In addition to the clinical evaluation of the right genu, the wound was clean without secondary infection. The swelling was reduced without pain, limited movement improved during activity, extremity could be moved and straightened. The patient did periodic evaluation every month and the swelling in the rightgenu was getting smaller. The patient also perfomedmedical rehabilitation (physiotherapy) of the lower extremities for mobilization routinely once a week. The anti-tuberculosis therapy continued for 12 months, with clinical knee joint returning to normal and the patient was able to walk without complaint (Figure 5).

\section{DISCUSSION}

Gonitis TB is extra-pulmonary TB in the knee joints due to hematogenous spread of distant primary infection, which can occur at any age, is common in children and adolescents more than adults. ${ }^{47}$ In this case, young female patient with an immunocompromised tendency with malnutrition and underweight (BW $40 \mathrm{~kg}$, height $152 \mathrm{~cm}$, BMI $17.3 \mathrm{~kg} / \mathrm{m}^{2}$ ) was prone to $\mathrm{M} . \mathrm{Tb}$ infection in bone and joint TB, where the lesions in this region are easy to replicate or multiply. The spread can be direct from osseous lesions of the epiphyseal (adult) or metaphysis (children), which causes erosion in the joint space and becomes arthritis. ${ }^{4,7}$

The infection process in gonitis $\mathrm{TB}$ occurs in synovial which begins at an early stage where there are atypical symptoms, including: 1) the thickening of the joint capsule, 2) the joints appear blurry and the gaps are slightly widened due to intra-articular effusion, or 3) osteoporosis in the bones around the joints due to hyperemia or increased blood flow in the tissue. ${ }^{7,9}$ The Triad Phemister of gonitis TB consisted of osteopenia of juxta-articular, peripheral bone erosion, and gradual narrowing of disc space. The condition will get worse if not being treated well, and it will destruct the joint space and ankylosis. ${ }^{6,10}$ The spread in the joints occurs as osteomyelitis through metaphysis and epiphyses or occurs directly in the joints which occurs in adolescence and adulthood. ${ }^{4,6,7}$

Gonitis TB is monoarthritis disease, but also occurs in multifocal lesions. Common complaints are fever, weight loss, malaise, night sweats, anorexia, and other pulmonary or extra-pulmonary TB symptoms. Early conditions of joint TB include the characteristics 


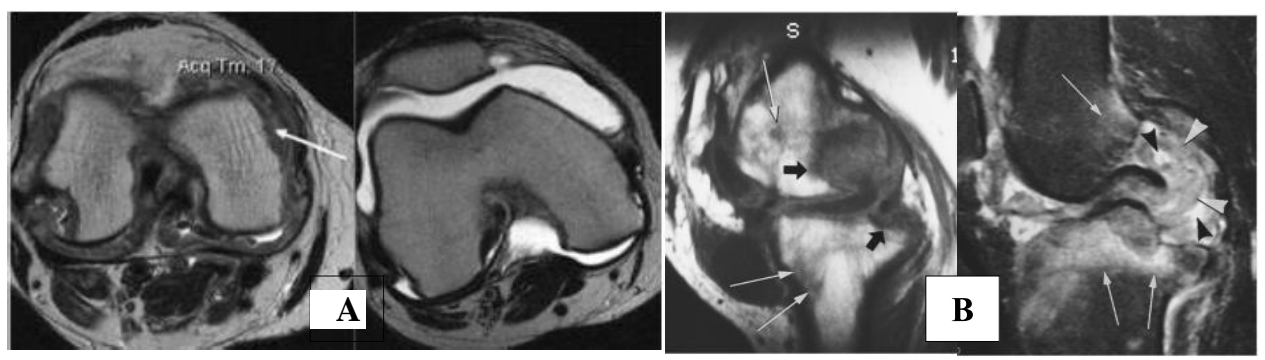

Figure 6. A) T2-weighted axial genu joint showed extensive synovial proliferation. Synovial attenuation indicates weak signal intensity. Genu joint showed a hyper-intense fluid effusion in the presence of fluid in the bursa, T1- weighted sagittal showed bone erosion and abnormal marrow signal. Joint TB which involved genu joint showed hypo-intense lesions (short arrow) and hypo-intense marrow (long arrow) bone erosions. B) T2-weighted sagittal genushowed signal intensity of synovial abnormalities and increased bone marrow signal intensity (arrow). Joint TB showed moderate abnormal signal intensity (white arrow) with little hyper-intense joint fluid (black arrow). ${ }^{11}$

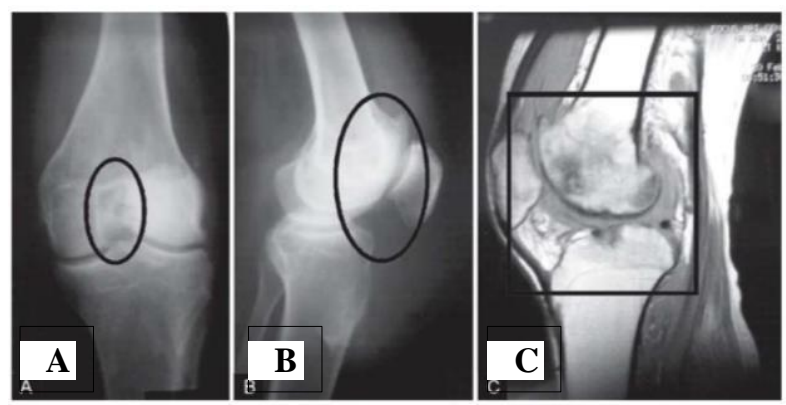

Figure 7. A). Radiological X-ray of left genu in an 18year-old male with a history of injury and increasing pain and stiffness of the left genu which did not respond to symptomatic treatment, showing smooth bone changes (small oval) and, B). patella-femoral sclerosis (large oval). C). Sagittal MRI showed infective changes affecting the epiphyseal region of the femur rather than the tibia. Genu biopsy examination revealed TB. ${ }^{5}$

such as arthritis: swelling, pain, limited movement, and redness without warm (cold abscess). ${ }^{10}$ The legs are in flexi position and can be accompanied by tenosynovitis, muscle spasm to muscle weakness, and resemble paralysis. The condition can be more advanced and severe with difficulty to move or lifting the joint, accompanied by pain. ${ }^{8}$

Symptoms of bone and joint TB are generally nonspecific and the clinical course is slow, it can delay the diagnosis. Other destruction findings include abscess, fistula, and joint deformities in advanced conditions. Gonitis TB can be accompanied by active pulmonary TB, but only about $50 \%$ of patients withbones and joints TB have chest X-rays according topulmonary TB, thus it can slow down the diagnosis of pulmonary TB. ${ }^{10}$

Chest radiological examination of extrapulmonary TB aims to determine and confirm the presence of pulmonary TB. ${ }^{10}$ Meanwhile, in Gonitis TB, the radiology is performed in the anterior-posterior (AP) and/or lateral position using computerized tomography (CT)-scan or MRI to determine the chronically occurring bone destruction. However, no radiological findings is pathognomonic for bone and joint TB. ${ }^{6,10}$

CT-scans of bone and joint TB are useful to evaluate the level of bone damage, soft tissue abscess, and enlargement of the surrounding soft tissue. Generally, conventional radiological examination is used as the initial imaging for the evaluation of $\mathrm{TB}$, but it can show negative results in early disease. Therefore, in suspected bone and joint TB, MRI examination should be considered. ${ }^{6}$ MRI can detect early changes, especially synovial thickening, periarticular soft tissue, and joint space. $^{4}$ MRI may also show earlier intra-osseous involvement compared with other imaging modalities. ${ }^{6}$

Bone abnormalities in gonitis TB are bone erosion or abnormal marrow signal intensity. Bone erosion is considered as a lesion causing disruption ofthe cortical contour of the bone, while abnormal marrow signal intensity is considered as poorly demarcated, diffuse or hypo-intense (T1-weighted) areas or as hyper-intense (T2) areas in the subchondral bone marrow (Figures $6 \&$ 7). ${ }^{11,9}$ In atypical clinical and plain radiology findings, MRI can assist the diagnosis (Figure 7). ${ }^{5}$

Other diagnostic of gonitis TB is by examining synovial fluid, by involvement of synovial infection. The synovial membrane becomes dense, edematous and tubercles are present. The synovial fluid is increasing, contains fibrin and an increase in the number of mononuclear cells $(\mathrm{MN})$. Initial infection begins in the metaphysis, resulting in an acute exudative infection with caseous necrosis. ${ }^{5}$ Non-hemorrhagic and cloudy synovial fluid is ranging from 10,000 to 20,000 cells/ul with $\mathrm{MN}$ leukocytes predominant. Aspiration for the infected joint fluid can be routine or standard 
examinations, and a culture test is recommended even if the culture is negative. Culture of synovial fluid is positive in $20-40 \%$ of the cases, M.Tb culture can be done to find the causative bacteria. The study conducted by $\mathrm{Gu}$, et al. showed that the GeneXpert MTB/RIF examination had $82 \%$ sensitivity and $100 \%$ specificity for diagnosis of bone and joint TB in 60 cases. ${ }^{12}$ The gold standard of diagnosis of gonitis TB is by synovial biopsy, with a positive result in $80 \%$ of the cases. A biopsy can show caseous granuloma, lymphocytes, and giant cells with caseosa, which are the characteristics of gonitis TB. ${ }^{13,14}$ Synovial fluid is often undiagnosed, biopsy examination and M.Tb culture of synovial and periarticular bone are required for the definitive diagnosis of TB gonitis. ${ }^{4}$

\section{Therapy}

TB in bones and joints is clinically divided into 4 stages: ${ }^{6,9}$

1) Synovitis stage

Soft tissue swelling (tissue swelling) without bone lesions, localized osteoporosis, USG examination can be confirmed, aspiration of synovial effusion for cytology examination, AFB staining examination and polymerase chain reaction (PCR). Surgical intervention is generally not required.

2) Early arthritis stage

Edge erosion (erosion of one or more lytic lesions in the bone or decreased joint space) with slight stiffness of the extremities. Clinical features with spasm and pain movement, MRI shows synovial effusion, osseous edema, and areas of bone destruction. Non-operative management can be given with analgesics, in case of failure to respond to nonoperative treatment, confirmation of the diagnosis is required. Synovectomy and debridement are performed with the aim of reducing the burden of the diseased tissue and confirming the diagnosis.

3) Advanced arthritis

Sub-perichondral cyst and joint spacenarrowing, may be accompanied by loss of motion. Joint margins are irregular and blurred, destruction of the bones in both joints, radiology X-ray findings on the extremities found erosions and joint space is reduced, limb shortening and deformity can complicate the condition.

4) Advanced stage of arthritis with dislocation

Joint destruction and immobile joint, ankylosis, and deformity. In advanced stage, erosions occur in the bones near the joints that are local or broad. Nearly half of the joints can become infected and bone erosion is seen on the surface of adjacent bones.
Damage in the form of a combination of signs of synovial and metaphysical infection and focus of epiphyseal destruction may occur. MRI examination is needed to assess intra-articular abnormalities including synovial thinning with abnormal signal intensity characteristics. Peri-artricular abnormalities in bone and soft tissue, including subchondralmarrow edema, cortical erosions, cellulitis, myocystis, soft tissue abscess, and skin ulceration/sinus formation. MRI can provide a picture of synovial proliferation. Synovial proliferation shows an intermediate to low picture of T2-weighted signal intensity. In contrasttreated patients, synovial hypertrophy can be seen. Marrow edema, osteomyelitis, cortical erosions, myositis, cellulitis, abscesses, and skin ulceration can be seen in bones and joints. ${ }^{9,11}$

Based on these stages, this case occurred in the second stage with an early arthritis stage which supported both clinical, physical, and radiological examination. Case management is also suitable for patients who do not respond to non-operative management by administering Mefenamic acid $500 \mathrm{mg}$ per 8 hours. Elective synovectomy and debridement are considered in order to reduce the burden of disease and confirm the diagnosis by orthopedic surgery. Early diagnosis of gonitis TB and the administration of effective therapy aim to save joints, based on findings at the beginning of the course of nonspecific disease. ${ }^{10}$ The basic principle of the treatment is by administering an effective and suitable antituberculosis regimentherapy for the treatment of bone and joint TB. A study by Sharma in 2009 recommended the treatment of bone and joint TB with a duration of 6-9 months, with some experts tended to use the treatment up to 9 months duration. ${ }^{5}$

However, based on the Indonesian Ministry of Health guidelines (2016), the treatment for bone andjoint TB is given for 9-12 months, and is recommended for 12 months according to clinical evaluation. ${ }^{15}$ In 2019, Indonesian Ministry of Health released a new guideline for administering anti-tuberculosis therapy in bone and joint TB with the standard regimen 2RHZE/10-16RH, which should be given 1 year to 18 months in special cases, especially if the clinicalcondition does not improve. ${ }^{10}$ Concomitant use of corticosteroids in the treatment of bone and joint diseases is not recommended. ${ }^{5}$

The duration of anti-tuberculosis therapy is given due to the weak penetration of the drug into bone and fibrous tissue and it is difficult to monitor the response 
to the treatment. Adequate combination therapy can be started without waiting for the culture results, if the histology and clinical features are consistent with the diagnosis of TB. Clinical response is best assessed through clinical indicators such as pain, constitutional symptoms, mobility, and neurological signs. ${ }^{10}$ Meanwhile, surgical therapy is generally planned for diagnosis and if necessary for drainage of an abscess that is unresponsive to the therapy or a large abscess to reduce pressure. ${ }^{4}$

Management of medical rehabilitation is considered after bone and joint surgery, though the surgical procedure is considered successful but the function of the movement depends on the post-operative rehabilitation program. ${ }^{16}$ After surgery, stiffness can occur due to the contracture to excessive scarring in the wound healing process, while functioning as a knee joint mobility and stability. Stiffness in the knee joint or limitation of movement is one of the complications. ${ }^{17}$

Other infections can also occur at the same time. Bacterial superinfection can mask diagnosis and presentation, and there have been a number of reports of coexisting infections due to Staphylococcus aureus and TB. ${ }^{4}$ In this case, from the radiological chest X-ray,there was an infiltrate in the right suprahillar and was supported by the results of sputum sensitivity culture which showed Pseudomonas aeruginosa which is a gram-negative bacteria. Based on this, the patient was treated according to the management of pneumonia with Ceftriaxone 1 gram per 12 hours as the cephalosporin beta-lactam antibiotic third-generation, initially beforethe results of the culture and sputum sensitivity examination were released. Antibiotics in this case were used as empirical antibiotics. From the laboratory evaluation, it obtained clinical and laboratory improvement, and there was a decrease in leukocytes from $12.3310^{3} / \mu 1$ to $9.5810^{3} / \mu 1$, thus the administration was up to 7 days. In giving empiric antibiotics that will respond well even though it does not match the results of culture and sensitivity, antibiotic administration can be followed by clinical evaluation. ${ }^{18}$

\section{CONCLUSION}

Gonitis TB is a hematogenous spread of M.Tb from an infection with a deep primary focus on the joint that is chronic progressive, and generally only affects one joint, accompanied by mild complaints that are getting worse with clinical symptoms of swelling, pain, limited movement, cold abscess, and redness. On physical examination, it can be found in the legs in a flexed position and in further conditions it is difficult to move and accompanied by pain. The diagnosis is based on radiological findings of bones and joints (AP/lateral, CT-scan or MRI). MRI examination is considered to assess early intra-articular abnormalities which include synovial thinning with abnormal signal intensity characteristics and can provide synovial proliferation. Synovial fluid examination can show an inflammatory process with MN leukocytes predominant. The gold standard of diagnosis in gonitis TB is synovial biopsy, with the appearance of caseous granulomas, lymphocytes, and giant cells with caseosa, which are the characteristics of joint TB. The management can be done by administering anti-tuberculosis and clinical monitoring, and completing anti-tuberculosis therapy for

12 months to show good results. In addition, it is recommended to examine chest $\mathrm{X}$-rays to determine the possibility of accompanying pulmonary TB and bacterial superinfection.

\section{REFERENCES}

1. Gambhir S, Ravina M, Rangan K, Dixit M, Barai S $\mathrm{B}$, J. Imaging in Extrapulmonary Tuberculosis. Int J Infect Dis 2016;

2. Held MFG, Hoppe S, Laubscher M, et al. Epidemiology of Musculoskeletal Tuberculosis in an Area with High Disease Prevalence. Asian Spine $J$ 2017;

3. Lidder S, Lang K, Haroon M, Shahidi M, ElGuindi, Magdi. Tuberculosis of the knee. Orthop Rev (Pavia) 2009;1.

4. Schlossberg D. Tuberculosis and Nontuberculous Mycobacterial Infections. Sixth Edit. Philadelphia, Pennsylvania: American Society for Microbiology (ASM) Press; 2011.

5. Sharma SK. Tuberculosis. Second Edi. New Delhi, India: Jaypee Brother Medical Pulblisher (P) Ltd; 2009.

6. Schaaf S, Zumla A. Tuberculosis: A Comprehensive Clinical Reference. Elsevier Inc.; 2009.

7. Fitzgerald DW, Sterling TR, Haas DW. Mycobacterium tuberculosis. In: Principles and Practice of Infectious Diseases. Elsevier Inc.; 2014. p. 2787-2818.

8. Shah M, Chida N. Extrapulmonary Tuberculosis. In: Handbook of Tuberculosis. Springer International Publishing Switzerland; 2017. p. 91- 118.

9. Thijn CJP, Steensma JT. Tuberculosis of the Skeleton: Focus on Radiology. 1st ed. SpringerVerlag Berlin Heidelberg; 1990.

10. Kementrian Kesehatan RI. Keputusan Menteri Kesehatan Republik Indonesia Nomor HK.01.07/Menkes/755/2019 tentang Pedoman Nasional Pelayanan Kedokteran Tatalaksana Tuberkulosis. 2019.

11. Lecomte AR, Ossiani M, Aliabadi P. Imaging of Infection. In: Imaging of Arthritis and Metabolic Bone Disease. 2019. p. 314-338. 
12. Wen H, Li P, Ma H, Lv G. Diagnostic accuracy of Xpert MTB / RIF assay for musculoskeletal tuberculosis: a meta-analysis. Dove Press Infect Drug Resist 2017;299-305.

13. Storm M, Vlok GJ. Musculoskeletal and spinal tuberculosis in adults and children. In: Tuberculosis: A Comprehensive Clinical Reference. Elsevier Inc.; 2009. p. 494-503.

14. Sanghvi DA, Iyer VR. MRI features of tuberculosis of the knee. Skelet Radiol 2009;267-273.

15. Kementrian Kesehatan RI. Peraturan Menteri Kesehatan Republik Indonesia No. 67 tahun 2016 tentang Penanggulangan Tuberkulosis. 2017.

16. MacLeay JM, Eades SC, Moore RM, Stokes AM. Diseases of the Musculoskeletal System. Tenth Edit. Elsevier Inc.; 2004.

17. Monika P, Anandh S. Effect of Functional Rehabilitation in Post Operative Knee Stiffness. Indian J Physiother Occup Ther - An Int J 2017;11(2):195.

18. Amin M, Koesoemoprodjo W, Hasan H, Marhana IA. Buku Ajar Paru 2019. Airlangga University Press; 2019. 